\title{
Peer Review post mortem. Bewertungen in akademischen Nachrufen
}

\author{
Julian Hamann, Forum Internationale Wissenschaft, University of Bonn
}

\begin{abstract}
Evaluations have a long history in academia. They do not only ascribe worth to individual achievements like article manuscripts and project proposals, but also to the academic personnel. Evaluations of academic persons are particularly apparent in academic obituaries, a genre that evaluates lifetime achievements of deceased academics. Drawing on a qualitative analysis of 216 obituaries that have been published in academic journals, this contribution reconstructs basic characteristics of evaluations of academic persons. The article highlights, first, typical practices of the evaluation of academic persons, second, temporal, national and disciplinary variations of selected evaluation criteria, and, third, it distinguishes modes of evaluation in which academics evaluate either themselves or others. Against this backdrop, the contribution develops an exploratory comparison with other academic and non-academic genres of the evaluation of persons: religious confessions, therapy, and biographies on scientists. The conclusion ties the findings back to general concepts of the sociology of valuation and evaluation.
\end{abstract}

\section{Zusammenfassung}

Bewertungen sind seit jeher ein zentraler Bestandteil der Wissenschaft. Sie schreiben nicht nur einzelnen Forschungsleistungen wie Artikelmanuskripten oder Projektanträgen Wert zu, sondern auch wissenschaftlichen Personen. Auf Personen bezogene Bewertungen werden besonders greifbar, wenn in Fachzeitschriften veröffentlichte Nachrufe die Lebensleistungen verstorbener Wissenschaftler würdigen. Der Aufsatz rekonstruiert auf der Grundlage einer qualitativen Analyse von 216 Nachrufen grundlegende Charakteristika dieser personenbezogenen Bewertungen. Erstens werden typische Praktiken herausgearbeitet, mit denen Personen in der Wissenschaft bewertet werden. Zweitens zeigt der Beitrag zeitliche, nationale und disziplinäre Variationen ausgewählter Bewertungskriterien auf. Drittens werden Modi der Selbst- und Fremdbewertung unterschieden. Vor diesem Hintergrund werden Nachrufe in einem explorativen Vergleich mit anderen akademischen und nicht-akademischen Genres personenbezogener Bewertung kontrastiert: der Beichte, der Therapie und der Wissenschaftlerbiografie. AbschlieBend werden die Befunde zurïckgebunden an allgemeinere Konzepte der Soziologie der Bewertung. 


\section{Einleitung}

Die Wissenschaft ist durchzogen von Bewertungen. So haben Wertzuschreibungen einen Einfluss auf die (Re-)Produktion wissenschaftlichen Wissens, indem sie Wahrheitsbehauptungen filtern und Qualitätsstandards legitimieren. Doch Bewertungen spielen nicht nur eine wichtige Rolle für wissenschaftliches Wissen. Sie sind auch von zentraler Bedeutung für die Wissenschaftler, die dieses Wissen produzieren und repräsentieren. Bei personenbezogenen Bewertungen geht es beispielsweise darum, Personen durch die Zuschreibung bestimmter Merkmale eine sozial verbindliche Anerkennung zuteilwerden zu lassen oder anhand zugeschriebener Eigenschaften zu sortieren, einzuordnen oder zu verorten (zu dieser Unterscheidung von Legitimierung und Kategorisierung als Aspekte von Bewertungsprozessen vgl. Lamont 2012).

Die Bewertung von Wissenschaftlerinnen ${ }^{1}$ findet in einer Vielzahl von Situationen und Genres statt. Die jeweiligen Bewertungskonstellationen konstituieren verschiedene Formen akademischer Subjektivität. Erstens werden Subjekte im akademischen Diskurs überhaupt erst durch Bewertungen in Erscheinung gebracht. Selbst vermeintlich stabile, weil kanonische Sprecherpositionen wie „Derrida“ werden durch Zuschreibungen immer wieder neu aktualisiert, wenn ausgehandelt wird, mit welchen Eigenschaften sie ausgestattet sind und wie sie sich zu anderen Positionen verhalten (Lamont 1987; Angermuller 2014). Zweitens werden wissenschaftliche Persönlichkeiten und Habitus sozial strukturiert, indem sie durch Bewertungen in Soziabilitätsregimen wie Gremien, Netzwerken und Karrieren konstituiert und diszipliniert werden. In diesen Regimen wird z. B. ausgehandelt, wie sich Wissenschaftler legitim inszenieren können (Beaufaÿs 2003). Zentrale Institutionen der Selbstbewertung sind, drittens, Curricula Vitae. Sie überführen die über einen Lebenslauf verteilten schulischen, beruflichen und akademischen Stationen und Verdienste in stringente und chronologische Biografien, die in Bewerbungssituationen wiederum Ausgangspunkt und Gegenstand von Fremdbewertungen werden (Miller und Morgan 1993; Dietz et al. 2000). Bürokratische Prozesse und organisationale Verfahren verflechten, viertens, akademische Subjekte mit institutionellen Posten wie dem des „Doktoranden“ oder der „Professorin“. Diese Form der Bewertung ist z. B. typisch für Berufungsverfahren. Sie hat ein legitimierendes Moment, weil institutionelle Posten Voraussetzung für eine sozial verbindliche Anerkennung in der Wissenschaft sind, und sie hat ein

\footnotetext{
${ }^{1}$ Im Sinne einer leichter lesbaren und doch geschlechtergerechten Sprache verwende ich im Folgenden abwechselnd das feminine und maskuline Genus. Damit soll nicht verschleiert werden, dass akademische Nachrufe vornehmlich Männer würdigen und von Männern verfasst werden. Von den 216 hier untersuchten Nachrufen wurden 93\% der Nachrufe von männlichen Wissenschaftlern geschrieben, und 95\% handeln von männlichen Wissenschaftlern.
} 
kategorisierendes Moment, weil solche Verfahren einen selektiven und sortierenden Charakter haben (Musselin 2009; Zimmermann 2000).

Diese Formen und Aspekte personenbezogener Bewertungen sind von zentraler Bedeutung in der akademischen Welt. Dennoch wurden bislang weder die Regeln noch die Kriterien der jeweiligen Bewertungsprozesse systematisch erforscht. Abseits vom situativen Bewertungswissen der involvierten Akteure ist nicht klar, welche Normen und welches Ethos zugrunde liegen, wenn Personen durch andere Personen bewertet und somit als Subjekte konstruiert, geformt, diszipliniert, institutionalisiert werden. Das Forschungsdesiderat hinsichtlich personenbezogener Bewertungen wird besonders deutlich im Vergleich zur relativ gut entwickelten Forschung zu Bewertungskonstellationen, die auf wissenschaftliche Produkte oder Organisationen ausgerichtet sind. Dazu gehören beispielsweise die Begutachtung von Projektanträgen für Förderorganisationen (Lamont 2009), die Beurteilung von Manuskripten für Fachzeitschriften (Hirschauer 2005) und nicht zuletzt die vergleichende Bewertung von Organisationen im Lichte wissenschafts- und bildungspolitischer Maßgaben (Sauder und Espeland 2009). Im Gegensatz zu diesen nicht auf Personen bezogenen Konstellationen blieben Bewertungen der Lebensleistungen holistisch gedachter Subjekte bislang weitgehend im Dunkeln. Dafür gibt es mindestens zwei Gründe: Die auf Personen und ihre Lebensleistungen anwendbaren Bewertungskriterien sind, erstens, so vielfältig, dass es wenige geeignete Daten gibt, in denen sich diese Bewertungsform empirisch in ihrer vollen Komplexität niederschlägt. Zweitens ist die Bewertung eines gesamten Lebens einer Person (nicht nur) in der Wissenschaft eine sensible Angelegenheit. Bestimmte Erhebungstechniken wie Befragungen oder Beobachtungen sind daher nicht immer anwendbar oder zielführend.

Der Beitrag behandelt akademische Nachrufe als ein besonderes Genre der personenbezogenen Bewertungen von Lebensleistungen. In den beiden folgenden Abschnitten (Abschn. 2; Abschn. 3) skizziere ich die für diese These relevanten Forschungsstränge zu akademischen Bewertungen und zu Nachrufen und stelle das Datenmaterial und die verwendete Methode dar. Im Hauptteil des Beitrages (Abschn. 4) arbeite ich zunächst grundlegende Praktiken personenbezogener Bewertungen in Nachrufen heraus, bei denen Wissenschaftler kategorisiert und verortet und ihre Lebensläufe anerkannt und legitimiert werden (Abschn. 4.1). Dann werden am Beispiel der Bewertungskriterien „Begabung“ und „Internationalität“ besonders auffällige zeitliche, nationale und disziplinäre Variationen von Bewertungen aufgezeigt (Abschn. 4.2). Anschließend rekonstruiere ich Selbst- und Fremdbewertungen als typische Modi der Bewertung in Nachrufen (Abschn. 4.3). Anhand eines Vergleichs mit anderen Genres personenbezogener Bewertungen zeigt der Beitrag schließlich, dass eine komparative und auch feldübergreifende 
Perspektive analytisch produktiv ist (Abschn. 5). Der letzte Abschnitt diskutiert diese Befunde und bindet sie zurück an allgemeine Konzepte einer Soziologie der Bewertung (Abschn. 6).

\section{Akademische Bewertungen, Positionierungen und die Analyse von Nachrufen}

Zu Fragen akademischer Bewertungen hat sich in den letzten Jahren ein breites Forschungsfeld entwickelt. Ein erster Forschungsstrang betont den zentralen Stellenwert von Evaluationen und Qualitätsurteilen in der Wissenschaft. Besondere Aufmerksamkeit erfahren aktuell numerische Ansätze, die beispielsweise Zitationen oder Publikationen erfassen und auf diese Weise Forschungsleistungen quantifizieren (vgl. das 2015 erschienene Themenheft „Der impact des impact factors" der Sozialen Welt). Peer-Review-Verfahren zur Bewertung wissenschaftlicher Produkte sind jedoch eine weitaus ältere Praxis. Mit ihnen werden nicht nur die Qualität, sondern auch die Passung der Produkte im Kontext von akademischen Journalen, Förderorganisationen oder Berufungsverfahren ermittelt. Soziologische Arbeiten beschäftigen sich mit der Reliabilität der hier gefällten Urteile (Bornmann und Daniel 2005), wägen potenziell dysfunktionale Effekte (Sandström und Hällsten 2008) auf der einen und die Funktionalität der Verfahren (Reinhart 2012; Hornbostel 1997) der anderen Seite ab, diskutieren unterschiedliche Definitionen von Leistung (Guetzkow et al. 2004; Tsay et al. 2003) und werfen schließlich die Frage auf, ob und wie Gutachterinnen überhaupt zu einem Konsens über „Qualität“ kommen (Hirschauer 2005; Lamont 2009).

Ein zweiter Forschungsstrang konzentriert sich auf die Kriterien und Normen, die den Hintergrund für akademische Bewertungen bilden. Untersuchungen in diesem Feld machen deutlich, dass numerische Maße wie Drittmittelinputs und Publikationsoutputs zwar Richtwerte für die Forschungsgovernance sein mögen, aber nicht ansatzweise die Vielfalt von Bewertungskriterien erfassen, die die akademische Welt durchdringen (vgl. Becher und Trowler 2001). Die Kriterien und Normen, auf die sich Wissenschaftlerinnen alltäglich beziehen, sind in der Regel zu ambivalent und unscharf, um verlässlich in statistischen Maßen erfasst werden zu können. Für eine Reihe von Bewertungskriterien - z. B. „Exzellenz“ (Lamont 2009), „Intellekt“" (Tsay et al. 2003), „Einschlägigkeit“ (Hirschauer 2005) oder „Originalität“ (Guetzkow et al. 2004) - ist gezeigt worden, dass sie jeweils situativ hervorgebracht werden, weil ihre Verhandlung in spezifische Kontexte wie Redaktionen von Fachzeitschriften, Gutachterpanels der Forschungsförderung oder die Aufnahmebedingungen von Fellowships eingebettet sind.

Beide Forschungsstränge leisten wichtige Beiträge zum Verständnis akademischer Bewertungen. Doch die bisher untersuchten Bewertungen sind, erstens, nur in Ausnahmefällen 
personenbezogen. In der Regel konzentriert sich die Literatur auf Bewertungen von wissenschaftlichen Produkten wie Manuskripten oder Projektanträgen und in geringerem Ausmaß auf Bewertungen von Organisationen wie Universitäten. ${ }^{2}$ Zweitens sind die untersuchten Bewertungen an sehr spezifischen Zielsetzungen und Passungsverhältnissen orientiert. Die je spezifische Ausrichtung von Bewertungsprozessen zeigt sich beispielhaft daran, dass Manuskripte immer auch mit Blick auf die Ausrichtung einer Fachzeitschrift und die Heftplanung beurteilt werden, dass bei Projektanträgen auch das Programm einer Forschungsförderung eine Rolle spielt und für Fellowships unter anderem die Grundsätze eines Stipendienprogrammes ausschlaggebend sind. Weil die bisher untersuchten Bewertungen im Wissenschaftsbereich als in spezifische Kontexte eingebettet konzipiert wurden, können die gewonnenen Einsichten nur in einem begrenzten Maße auf Bewertungen ganzheitlich gedachter akademischer Biografien und wissenschaftlicher Lebensleistungen übertragen werden. Zentrale Praktiken, Kriterien und Modi der personenbezogenen Bewertung in der Wissenschaft bleiben daher unterbelichtet; wichtige Fragen zur Bewertung von Personen durch Personen, z.B. zu den Folgen, die Bewertungen für Bewertete und Bewertende haben, werden vernachlässigt.

Genau diese Perspektiven werden in einem dritten Forschungsstrang verfolgt, der Studien zu Positionierungen von Wissenschaftlern umfasst. Die hier untersuchten Selbst- und Fremdzuschreibungen von Positionen können als Formen der Kategorisierung, Sortierung und Verortung von Wissenschaftlerinnen beziehungsweise als Formen ihrer sozialen Anerkennung und Legitimierung verstanden werden. Wie die Bewertung selbst ist auch diese Positionierungsarbeit von kulturellen, institutionellen und sozialen Kontexten geprägt: So haben z.B. spezifische soziopolitische Umstände in Frankreich den Aufstieg existenzialistischer Positionen begünstigt (Baert 2011), werden der Sprecherposition „Derrida“ in verschiedenen Ländern unterschiedliche Eigenschaften zugewiesen (Lamont 1987) und variieren die Positionierungen von Ökonomen je nach politischen, medialen oder wirtschaftlichen Arenen (Maeße 2015).

Andere Arbeiten in diesem Forschungsstrang konzentrieren sich weniger auf die Tatsache, dass Positionierungen von den sie umgebenden Kontexten strukturiert werden. Sie heben hingegen hervor, dass es für Wissenschaftler ein praktisches Problem ist, Sprecherpositionen zu definieren und zu besetzen. Diese Herausforderung besteht sowohl bei der Grenzarbeit zwischen wissenschaftlichen und nichtwissenschaftlichen Wissensfeldern (Gieryn 1999) als auch

\footnotetext{
${ }^{2}$ Soweit ich sehe, haben einzig Lewis (1998) und Tsay et al. (2003) mit ihren Untersuchungen von Empfehlungsschreiben ein akademisches Bewertungsgenre analysiert, das zumindest teilweise eine biografische Perspektive hat. Interessanterweise versäumen es aber beide Studien, die von ihnen herausgearbeiteten Bewertungspraktiken und -kriterien dahingehend zu hinterfragen, ob es in ihnen auch um die Bewertung der akademischen Lebensleistungen von Personen gehen könnte.
} 
in den ineinander verschränkten Wissen-Macht-Komplexen der Wissenschaft selbst (Angermuller 2013, 2014). Für Selbstbewertungen spielen Selbstkonzepte und -narrative eine besondere Rolle (Gross 2002; Bamberg 2011). Es ist naheliegend, davon auszugehen, dass Positionierungen weder in der Form von Kategorisierungen noch von Legitimierungen konfliktfrei ablaufen. Sie sind daher auch als Gegenstand symbolischer Kämpfe beschrieben worden, in denen Wissenschaftlerinnen aufgrund ihrer Kapitalausstattung präreflexive, habituell vermittelte Strategien verfolgen (Bourdieu 1992; Beaufaÿs 2003).

Ob Positionierungen nun als in Feldern strukturierte Kämpfe oder als alltäglich zu lösendes Problem verstanden werden: Die im dritten Forschungsstrang versammelten Studien zeigen, dass bei der Definition und Zuschreibung von Subjektpositionen Prozesse der Selbst- und Fremdkategorisierung sowie der sozial verbindlichen Anerkennung von Personen ineinandergreifen. So ermöglichen diese Arbeiten wichtige Einsichten in die Entstehung von Biografien, Identitäten und Subjektpositionen. Obwohl damit zentrale Aspekte personenbezogener Bewertungskonstellationen beleuchtet werden, steht die Literatur zu Positionierungen in der Wissenschaft bisher noch nicht in einem systematischen Austausch mit der Bewertungssoziologie. Es ist davon auszugehen, dass die Untersuchung von Fremd- und Selbstpositionierungen Erkenntnisse für die personenbezogene Bewertung akademischer Subjekte bereithält. $\mathrm{Zu}$ fragen wäre z. B., erstens, nach zentralen Praktiken, über die Selbst- und Fremdbewertungen von Wissenschaftlerinnen vorgenommen werden. Von Interesse ist, zweitens, ob sich allgemeinere Kriterien für akademische Selbst- und Fremdbewertungen identifizieren lassen und inwiefern diese Kriterien über zeitliche, nationale und disziplinäre Kontexte hinweg gelten. Drittens stellt sich die Frage nach dem Zusammenspiel von Selbst- und Fremdbewertungen.

Der vorliegende Beitrag geht diesen Fragen nach. Die Möglichkeit der Untersuchung dieser Fragen hängt nicht zuletzt von einem hierfür geeigneten Datenmaterial ab. Im Rahmen von Prüfungen und Evaluationen einzelner Leistungen mögen Bewertungen im akademischen Betrieb penibel dokumentiert sein. Doch auch wenn die Bewertung holistisch gedachter Personen im akademischen Alltag nicht weniger allgegenwärtig ist, sind die entsprechenden Praktiken, Kriterien und Modi der Wertzuschreibung selten offen dokumentiert und empirisch zugänglich. Daher greift mein Beitrag auf ein Genre zurück, in dem akademische Lebensleistungen explizit und öffentlich bewertet werden: akademische Nachrufe. Generell stützt sich die Forschungsliteratur zu diesem Thema vor allem auf Nachrufe in Zeitungen und nimmt hier insbesondere zwei Perspektiven ein: Sozialstrukturell argumentierende Ansätze begreifen Nachrufe als eine Art kollektives Gedächtnis und fragen danach, ob sie bestimmte Bevölke- 
rungsgruppen bevorzugt thematisieren und damit erinnern (Fowler 2007), oder gehen der sozialen Selektivität von Nachrufen bezüglich der Schichtzugehörigkeit (Fowler und Bielsa 2007) und dem Geschlecht der Verstorbenen nach (Rodler et al. 2001). Sozialkonstruktivistische Ansätze begreifen demgegenüber die Nachrufe vor allem als Orte der Repräsentation und Symbolisierung von Identitäten (Bonsu 2007; Long 1987).

Wie die Forschung zu Nachrufen in Zeitungen lässt sich auch die Literatur zu in wissenschaftlichen Fachzeitschriften veröffentlichten Nachrufen in sozialstrukturelle und sozialkonstruktivistische Ansätze unterteilen. So stehen sozialstrukturell informierten Studien, die z. B. die soziale Zusammensetzung der in Nachrufen erinnerten Ingenieurselite untersuchen (Gispen 1994), eine Reihe von sozialkonstruktivistischen Arbeiten gegenüber, die Nachrufe als möglichen Spiegel für bestimmte Aspekte der akademischen Welt verstehen, beispielsweise für die Dominanz der US-amerikanischen Wissenschaft (Tight 2008), für Praktiken der Ehrung und Wertung (Echterhölter 2012), für informelle Quellen intellektueller Reputation (Macfarlane und Chan 2014) oder für den karriererelevanten Imperativ der Internationalität (Hamann und Zimmer 2017). Mit ihrem Fokus auf Bewertungen in Nachrufen ordnet sich die hier vorgelegte Analyse in die konstruktivistische Sichtweise ein. Eine Verknüpfung mit den sozialstrukturellen Merkmalen der Autoren der Nachrufe und der posthum gewürdigten Wissenschaftler steht noch aus.

\section{Daten und Methode}

Vor dem Hintergrund des Forschungsstandes bieten sich Nachrufe als Datenmaterial an, um Bewertungen akademischer Lebensleistungen zu untersuchen. Im Gegensatz zu anderen Bewertungsgenres thematisieren Nachrufe ihrem Anspruch nach die gesamte akademische Person, indem sie ihre Lebensleistung bilanzieren. Dieses Urteil wird, zumindest rhetorisch, von einem Autor im Namen der Fachgesellschaft gefällt. Der Forschungsstand zu akademischen Nachrufen zeigt bereits, dass Nachrufe im Unterschied zu anderen Bewertungen auf die feierliche Erinnerung akademischer Verdienste und Lebensleistungen ausgerichtet sind. Das ehrliche und kritische Urteil ist hier nicht immer gefragt, sodass es auch nicht überraschen kann, wenn negative Aspekte, wie z.B. eine Kritik an der verstorbenen Person und ihrer Arbeit bzw. Brüche im Lebenslauf, ausgeklammert bzw. im Nachhinein oft umgedeutet werden. Nichtsdestotrotz zeigen bisherige Studien, dass Nachrufe ein performativer Ausdruck von im akademischen Feld akzeptierten Bewertungspraktiken und -kriterien sind (Tight 2008; Hamann 2016). 
Das der vorliegenden Untersuchung zugrundeliegende Sample von 216 Nachrufen habe ich aus einer Gesamtpopulation von 841 Nachrufen aus zentralen Fachzeitschriften und Publikationen von Fachverbänden zusammengestellt. Um zu gewährleisten, dass das Sample eine hinreichende empirische Vielfalt aufweist, in der dann grundlegende Elemente identifiziert werden können, habe ich zwei Samplingstrategien kombiniert. Der Strategie des stratifizierten Samplings folgend wurden zunächst verschiedene Subpopulationen eingeführt (vgl. Patton 2002). Die Nachrufe im Sample wurden in verschiedenen Zeiträumen, Ländern und Disziplinen veröffentlicht (1960er-, 1980er-, 2000er-Jahre; Großbritannien, USA, Deutschland; Physik, Soziologie, Geschichtswissenschaft). Darauf habe ich mit einer Strategie des gerichteten Samplings aufgebaut und jeder Subpopulation die gleiche Anzahl von Fällen zugeteilt (vgl. Emmel 2013). Die 216 Nachrufe verteilen sich also zu je 72 auf drei Länder, drei Disziplinen und drei Jahrzehnte. Auch bei dieser Aufteilung ging es darum, für die qualitative Studie eine empirische Vielfalt herzustellen, anhand derer verbindende Elemente und grundlegende Charakteristika identifiziert werden können.

Um die Bewertung von Lebensleistungen zu rekonstruieren, habe ich die Texte aus dem Sample in zwei Durchgängen kodiert. In einem ersten Durchgang wurden durch eine eng am empirischen Material orientierte inhaltsgeleitete Kodierung verbreitete oder charakteristische Bewertungsdynamiken und -dimensionen markiert. Im zweiten Durchgang, dem axialen Kodieren, wurden diese Kodes verknüpft oder verfeinert, bis grundlegende Praktiken, Kriterien und Modi der Bewertung identifiziert werden konnten und ein Punkt der Sättigung erreicht war, an dem keine neuen Unterthemen oder Beziehungen mehr rekonstruiert werden konnten (vgl. Strauss und Corbin 1990). Dieses Verfahren hat zu den Bewertungspraktiken, -kriterien und -modi geführt, auf die sich die folgende Analyse bezieht.

\section{Die Bewertung akademischer Lebensleistungen in Nachrufen: Praktiken, Kriterien, Modi}

Um grundlegende Charakteristika der personenbezogenen Bewertung herauszuarbeiten, konzentriert sich die Analyse, erstens, auf typische Bewertungspraktiken (Abschn. 4.1). Sie illustrieren, wie Wissenschaftlerinnen durch Kategorisierungen verortet und sortiert und wie ihre Lebensläufe anerkannt und legitimiert werden. Zweitens untersucht der Beitrag Bewertungskriterien, an denen akademische Lebensleistungen gemessen werden (Abschn. 4.2). Um auf- 
fällige zeitliche, nationale und disziplinäre Variationen von Bewertungen in Nachrufen beispielhaft aufzuzeigen, liegt der Fokus auf Kriterien, die nicht gleichmäßig im gesamten Sample verteilt sind. Drittens werden Bewertungsmodi der Selbst- und Fremdbewertung unterschieden (Abschn. 4.3). Damit wird gezeigt, dass Bewertungen in Nachrufen nicht allein auf die Verstorbenen beschränkt sind, sondern auch Selbstbewertungen der Autoren umfassen.

\subsection{Bewertungspraktiken}

Bei der Bewertung akademischer Lebensleistungen können verschiedene Praktiken unterschieden werden. Besonders charakteristisch ist, erstens, dass Verstorbene mit Bezug auf institutionelle Posten und Funktionen einerseits sowie Mitgliedschaften in Arenen spezialisierten Wissens andererseits kategorisiert und sortiert werden. Trotz der Neigung des Genres zu einer positiven Sicht auf die Lebensleistung müssen in Nachrufen, zweitens, auch biografische Brüche oder fachliche Kritik in die Erzählung integriert werden. Unter Bewertungsaspekten ist gerade interessant, wie diese negativen Aspekte als Teil einer kohärenten Gesamterzählung eines verdienstvollen Lebens anerkannt und legitimiert werden.

Bewertungspraktiken der Kategorisierung kommen zum Einsatz, wenn Wissenschaftlerinnen sich und andere über Merkmals- und Leistungszuschreibungen in diversen akademischen Kontexten verorten und sortieren. Worüber arbeitet die Person, die ich auf der letzten Konferenz kennengelernt habe? Wer vertritt meinen Forschungsbereich im neu erschienenen Handbuch? Fragen wie diese gehören zum akademischen Alltag, und die gegenseitige Verortung von Wissenschaftlern endet nicht mit dem Tod. Nachrufe referieren vor allem auf zwei Kontexte, anhand derer sie ihre Bewertungsobjekte kategorisieren. Sie platzieren Verstorbene, erstens, in Arenen spezialisierten wissenschaftlichen Wissens und kategorisieren sie dabei beispielsweise als Experten besonderer Forschungsgebiete oder Mitglieder bestimmter Communities. Zweitens werden Verstorbene in institutionellen Kontexten platziert, wodurch sie hinsichtlich ihres Status und ihrer institutionellen Verdienste und Funktionen bewertet werden (vgl. zu diesen Positionierungslogiken Angermuller 2013; siehe auch Hamann 2016). Die Unterscheidung dieser beiden Positionierungskontexte ist heuristisch-analytischer Natur. Empirisch können Bewertungspraktiken der Kategorisierung durchaus auf mehrere Kontexte gleichzeitig verweisen. Gerade für die Art von spezialisierter Fachgemeinschaft, die von akademischen Nachrufen adressiert wird, stellt z. B. eine Professur am Department of Sociology der Universität Chicago nicht nur die Bezeichnung eines institutionellen Status dar, sondern auch 
eine Kategorisierung mit Bezug auf ein besonderes wissenschaftliches Wissen (in diesem Fall der sogenannten „Chicago School“; vgl. Abbott 1999).

Dennoch lassen sich Zuschreibungen von Positionen in den beiden Kontexten analytisch differenzieren. In Arenen wissenschaftlichen Wissens werden Verstorbene beispielsweise als Angehörige bestimmter Schulen, Communities oder Forschungsbereiche kategorisiert. Das kann über eine generelle Einordnung vollzogen werden, die den Verstorbenen z.B. als „Nestor der deutschen Geschichtswissenschaft“ (Broszat 1984, S. 689) oder als „Hauptfigur der internationalen Physik“ (Close und Gal 2006, S. 6) ${ }^{3}$ verortet. Verstorbene können aber auch anhand spezifischer akademischer Leistungen markiert werden. Dazu gehören die Weiterentwicklung des empirischen Kenntnisstandes in einem Feld, z. B. wenn ein Verstorbener „die Epoche Anton Fuggers aus einer Sicht erhellte, die man bisher nicht geahnt hatte“ (Kellenbenz 1969, S. 286), sowie methodische Verdienste, beispielsweise wenn ein Verstorbener „bekannt für seine Arbeit zu Techniken der Netzwerkanalyse“ war (Hargens und Gieryn 1988, S. 572). Charakteristisch sind außerdem relationale Positionierungen. Sie setzen die Verstorbene mit kanonischen Autoren des jeweiligen Fachbereichs in Beziehung und bemühen dazu etwa Figuren wie Einstein (Brïche 1960, S. 257), Mommsen (Morris 1970, S. 147) oder Weber (Bonnell 1991, S. v). Zusätzlich dienen auch Personen aus dem Umfeld der Verstorbenen als Referenzfigur, wenn beispielsweise Kollegen (Lynch 2011, S. 930) oder Lehrer (Lemert und Piccone 1981, S. 163) in die Wertzuschreibung einbezogen werden. Generelle Kategorisierungen, konkretere Leistungszuschreibungen und relationale Positionierungen sind typische Praktiken, mit denen Verstorbene in Kontexte spezialisierten wissenschaftlichen Wissens einsortiert werden.

Bewertungspraktiken der Kategorisierung beziehen sich, zweitens, auf institutionelle Kontexte. Dazu gehören vor allem zahlreiche Verweise auf einen bestimmten institutionellen Status wie z.B. Forschungsprofessor (Close und Gal 2006, S. 5), Dekan (Kellenbenz 1969, S. 286) oder Rektor (Wehler 1985, S. 150). Hierbei werden Wissenschaftler nicht anhand ihrer Zugehörigkeit zu bestimmten Fachgemeinschaften oder ihrer Beiträge zu Diskussionen innerhalb dieser Gemeinschaften sortiert, sondern anhand ihres Status in der institutionellen Ordnung der akademischen Welt. Auf institutionelle Kontexte verweisen weiterhin nicht nur Positionen in der universitären Hierarchie, sondern auch in Fachverbänden (Rehberg 2003, S. 821), Herausgebergremien (Foster 2013, S. 3) oder Beiräten (Hargens und Gieryn 1988, S. 573). Bewertungen in Form institutioneller Verortungen unterstreichen „die besondere Bedeutung

\footnotetext{
${ }^{3}$ Zur besseren Lesbarkeit wurden alle englischsprachigen Zitate aus den Nachrufen vom Autor übersetzt.
} 
grundlegender organisatorischer Arbeit im Sinne einer Disziplin“(Blumer 1967, S. 103). Während Positionierungen in institutionellen Kontexten auf Status abzielen, bezeichnen Positionen in Räumen spezialisierten wissenschaftlichen Wissens die fachliche Reputation des Verstorbenen. Institutioneller Status und fachliche Reputation sind auch zu Lebzeiten zentrale Referenzen für die Kategorisierung von Wissenschaftlern. Es ist daher kein Zufall, dass diese auch für Nachrufe charakteristischen Referenzkontexte den akademischen und institutionellen Kapitalsorten entsprechen, die wissenschaftliche Felder strukturieren (Bourdieu 1992).

Neben der Kategorisierung von Wissenschaftlerinnen sind in Nachrufen auch solche Bewertungspraktiken typisch, die der Legitimierung von Wertzuschreibungen dienen. In der Regel geschieht dies, indem Erfolge und Verdienste im Rahmen einer biografischen Gesamterzählung als nachvollziehbar, einleuchtend oder plausibel präsentiert werden. Eine solche narrative Integration leisten z. B. Erzählungen einer schicksalshaften Bestimmung, nach der der Verstorbene stets zur rechten Zeit am rechten Ort war. Dabei werden Motive wie die Rede von einer „besonderen Fügung“ bemüht (Kellenbenz 1969, S. 283), und es werden Lebensgeschichten erzählt, die sich „fast wie ein Märchen“ lesen (Kopal 1972, S. 259). Selbst in Nachrufen erscheinen solche Darstellungen jedoch nicht immer als realistisch. Die Autoren der Nachrufe stehen dann vor der Herausforderung, auch negative Aspekte wie z.B. biografische Brüche oder fachliche Kritik glaubhaft in eine positive Gesamterzählung einzuordnen.

Die Schilderung negativer Aspekte erfordert viel Fingerspitzengefühl von den Autorinnen. Zwar besteht in akademischen Nachrufen grundsätzlich die Tendenz, externe Rückschläge, Brüche oder Kritik zu verschleiern (Macfarlane und Chan 2014; Tight 2008). In der Regel werden aber biografische Schwierigkeiten von Wissenschaftlern - von persönlichen Krisen bis zur fehlenden Finanzierung - gar nicht erst erwähnt. An anderer Stelle habe ich diese Auslassung als Ergebnis einer durch informelle Konsekrationsregeln des Genres ausgeübten Zensur interpretiert (Hamann 2016). Nachrufe präsentieren legitime akademische Subjekte vorwiegend als intrinsisch motivierte handlungsmächtige Akteure, die ihr Leben eigenmächtig kontrollieren und lenken.

Nichtsdestotrotz finden sich auch in akademischen Nachrufen Abweichungen vom dominanten Muster der Erfolgsgeschichte. Die Integration dieser Abweichungen in eine euphemistische Gesamterzählung ist aus bewertungssoziologischen Gesichtspunkten interessant. Wie geht ein Genre, das auf die feierliche Ehrung verdienstvoller Lebensläufe ausgerichtet ist, beispielsweise damit um, wenn bei dem Geehrten akademische Anerkennung zunächst ausbleibt und Erfolge auf sich warten lassen? Eine Möglichkeit, auch weniger glanzvolle Kapitel 
der Lebensgeschichte zu plausibilisieren, ist, sie im Nachhinein zu rationalisieren. Ausbleibende Anerkennung oder anfängliche Misserfolge werden dann ex post als Missverständnisse interpretiert, die sich im weiteren biografischen Verlauf auflösen. Ein Buch hat seine Wirkung dann nur noch nicht entfalten können, weil „die Bücher, die darauf aufbauen, noch nicht geschrieben wurden“ (Morris 1970, S. 149). Frühe Misserfolge werden damit erklärt, dass ein Wissenschaftler eben „erst spät zu hohen Ehren gekommen“ ist (Stein 1973, S. 139). Ganz ähnlich können Autoren von Nachrufen als finales Korrektiv wirken, z. B. wenn sie hervorheben, dass die Leistungen eines Verstorbenen „weit umfangreicher waren, als gemeinhin angenommen wird“ (Lynch 2011, S. 928), oder dass das Werk eines Verstorbenen „bisher zu wenig bekannt“ und seine Wirkung „ungebührlich beschränkt“ ist (Luckmann 1961, S. 768).

Eine zweite Möglichkeit, biografische Brüche zu plausibilisieren, ist ihre Externalisierung. Brüche und Krisen werden externen Faktoren zugeschrieben und liegen dann nicht mehr in der Verantwortung der Verstorbenen. So können institutionelle Umstände der Grund dafür sein, dass ein Wissenschaftler „sein Forschungsinteresse in ein neues Feld verlegt hat“" (Richards 1962, S. 1), während die Abweichung von einem kohärenten Forschungsprofil zu einer „Verschiebung wissenschaftlicher Interessen“ wird, ermöglicht durch die neue Anstellung an einem Observatorium (Kopal 1972, S. 261). Auch politische Umstände dienen dazu, Brüche zu externalisieren, beispielsweise wenn Wissenschaftler „die politische Bedeutung“ eines Themas erkannten und „deswegen begannen, es zu erforschen“ (Liddington 1984, S. 214), oder wenn der Zusammenbruch der deutschen Großmacht nach 1945 als Grund dafür angeführt wird, dass sich der Verstorbene ,,von einer isolierten Nationalgeschichte zu einer vergleichenden europäischen Geschichte“" wandte (Wehler 1985, S. 145). Gemein ist diesen Praktiken, dass die Gründe für nicht-lineare und erklärungsbedürftige Biografieverläufe in zeitbedingte Umstände ausgelagert werden. Wenn mangelnde Anerkennung auf einer Fehldeutung tatsächlicher Leistungen beruht und thematische Um- oder Desorientierungen zu durch äußere Gründe verursachten „Verschiebungen“ werden, können Lebensläufe trotz dieser Brüche und Krisen legitimiert und anerkannt werden.

Externalisierungen und ex-post-Rationalisierungen ermöglichen es demnach, auch Brüche und eine mangelnde Anerkennung in ein gerichtetes biografisches Erfolgsnarrativ zu integrieren. Lebensläufe erscheinen dann am Ende doch als weitgehend fehler- und widerspruchsfreie Biografien. Besonders an diesem Beispiel wird deutlich, dass Bewertungen in akademischen Nachrufen keinen dokumentarischen Charakter haben. Die in ihnen vorgenommenen Zuschreibungen sind hochselektiv und konstituieren sehr spezifische biografische Por- 
traits (zum Aspekt der Subjektkonstitution vgl. Hamann 2017). Der mangelnde dokumentarische Charakter von Nachrufen sollte jedoch nicht darüber hinwegtäuschen, dass das Genre dennoch sehr reale Wirkungen entfalten kann. Als Konsekrationsinstanz verleiht es Biografien eine Weihe, die für die Nachwelt eine soziale Verbindlichkeit im Sinne einer Vorbildlichkeit aufweist. So scheint es beispielsweise denkbar, dass die glanzvolle Konstruktion biografischer Idealbilder und die Anrufung akademischer Subjekte als intrinsisch motivierte, handlungsmächtige Akteure einen Aufforderungscharakter entfalten, der als Anreiz zur Nachahmung fungiert, aber auch Druck auf Wissenschaftlerinnen ausübt, ihre eigene Lebenspraxis an den Referenzkontexten und Plausibilitäten auszurichten, die in Nachrufen angerufen werden.

\subsection{Bewertungskriterien}

Während man anhand von Bewertungspraktiken zeigen kann, wie Wertzuschreibungen vorgenommen werden, lässt sich mit Blick auf die Bewertungskriterien genauer untersuchen, welche Werte zugeschrieben werden. Der Stellenwert von Bewertungskriterien kann je nach disziplinären, zeitlichen oder nationalen Kontexten variieren. Im Folgenden behandele ich zwei Bewertungskriterien, deren Varianz in diesen Dimensionen im Untersuchungssample besonders offensichtlich ist: „Begabung“ und „Internationalität“.

Ein vor allem in deutschen Nachrufen verbreitetes und somit national spezifisches Kriterium für akademische Biografien ist „Begabung“. Mit dieser Zuschreibung werden scheinbar angeborene Talente markiert oder Fähigkeiten naturalisiert. Verstorbenen Professoren wird beispielsweise ein ,untrügliche[r] Blick“ (Wehler 1985, S. 147) oder ein ,genuines Verständnis“ (Gollwitzer 1967, S. 297) für ihre Gegenstände attestiert. In eine ähnliche Richtung zielen auch zahlreiche Verweise auf eine „Berufung“ als Forscherin (Fouquet 2000, S. 2). Den Nachrufen zufolge zeigen sich Talente, Begabungen und Berufungen schon in frühen Lebensstadien. So kündigen sich in den biografischen Erzählungen bereits in der Dissertation Fragestellungen an, die einen Soziologen dann ,sein Leben lang beschäftigen“ (Luckmann 1961, S. 769), während eine Historikerin seit dem ,5. Semester fasziniert“ war von ihrem Fachgebiet (Fouquet 2000, S. 1) und ein Physiker seit seiner Schulzeit von den Röntgenstrahlen „nicht mehr losgelassen" wurde (Brüche 1960, S. 257).

Die von den Autoren hervorgehobene Bedeutung des Kriteriums „Begabung“ verweist auf in Deutschland noch immer einflussreiche Vorstellungen einer ganzheitlichen Bildung durch Wissenschaft und der wechselseitigen Durchdringung von Individuum und Forschungsgegenstand (Hamann 2014). Dieser kulturelle Hintergrund legt nahe, dass die akademische 
Leistungsfähigkeit schon immer in der Persönlichkeit eines Wissenschaftlers angelegt ist und nur noch erweckt werden muss. Im angloamerikanischen Raum ist das Kriterium „Begabung“ hingegen weniger verbreitet. Ein Grund hierfür kann die meritokratische Ideologie des „American Dream“ sein (McNamee und Miller 2004). Dieser Erzählung nach ist Erfolg prinzipiell für jede und jeden möglich, solange sie oder er nur hart genug dafür arbeitet. Akademische Verdienste können dementsprechend weniger durch die Zuschreibung einer angeborenen „Begabung" plausibilisiert werden. Tatsächlich werden in US-amerikanischen Nachrufen eher Bewertungskriterien wie Fleiß und Hingabe verwendet, etwa wenn der ,unermüdliche Arbeitseifer“ (Blumer 1967, S. 103) oder „Durchhaltevermögen und harte Arbeit“ (Gombrich 1981, S. 348) hervorgehoben werden.

Ein Bewertungskriterium, dessen Bedeutung je nach Disziplin und im Zeitverlauf variiert, ist „Internationalität““. So nachdrücklich wie in keiner anderen der untersuchten Disziplinen werden Wissenschaftlern in der Physik internationale Verdienste, Kosmopolitismus und internationale Mobilität zugeschrieben. Physiker werden beispielsweise anhand ihrer „,internationalen Orientierung“ (Peacock und Christiansen 2005, S. 78) oder ihrer Forschungsaufenthalte im Ausland (Kopal 1970, S. 147) bewertet. Die geringste Bedeutung hat das Kriterium der Internationalität in geschichtswissenschaftlichen Nachrufen; die Soziologie nimmt eine Mittelposition ein. Neben diesen disziplinären Variationen ist ein deutlicher Anstieg von $\mathrm{Zu}-$ schreibungen der „Internationalität“ im Zeitverlauf zu erkennen. Über alle Disziplinen hinweg wird es immer wichtiger, dass Wissenschaftler ,auf der internationalen Bühne respektiert“ (Roberts 2007, S. 613), ja sogar in ihrer „Wirksamkeit längst transnational“ waren, „ehe die entsprechenden Begriffe in Mode kamen“ (Rehberg 2003, S. 821).

Akademische Strukturen, Personen und Praktiken sind immer stärker über Ländergrenzen hinweg vernetzt (Huang et al. 2014). Die Rekonstruktion von „Internationalität“ als Bewertungskriterium ergänzt diesen Befund der Hochschulforschung, indem der Blick darauf gelenkt wird, dass „Internationalität“ nicht nur ein als gegeben anzunehmendes Phänomen, sondern auch eine diskursiv konstruierte Zuschreibung ist (dazu vertiefend Hamann und Zimmer 2017). Die steigende Bedeutung dieses Kriteriums im Zeitverlauf verweist darauf, dass eine hohe globale Mobilität für die Würdigung akademischer Lebensleistungen zunehmend wichtiger wird. Mögliche Gründe für die unterschiedliche Bedeutung von „Internationalität“ in der Physik und der Geschichtswissenschaft können z. B. disziplinenspezifische Kommunikationsstrukturen sein. Während die Naturwissenschaften mit englischsprachigen Publikationen längst in internationalen Kommunikationszusammenhängen operieren, haben nationalsprachliche 
Publikationen in den meisten Geisteswissenschaften weiterhin eine hohe Bedeutung (Alexander von Humboldt-Stiftung 2009; Dávidházi 2014). Dass sich diese Differenzen sogar in Nachrufen niederschlagen, relativiert Diagnosen einer multi- oder transdisziplinären Wissenschaft, indem sie an die Bedeutung erinnern, die Disziplinengrenzen für die Bewertungskriterien haben (vgl. dazu auch Lamont 2009; Huutoniemi 2012). ${ }^{4}$

\subsection{Bewertungsmodi}

Zwischen den Autoren als bewertenden Instanzen und den Verstorbenen besteht oft eine enge Beziehung, die auch als solche offengelegt wird. Nachrufe werden zumeist explizit für ehemalige Lehrerinnen, Weggefährten oder Kolleginnen geschrieben. Diese Art der personalisierten Widmung kann sich auf die in den beiden vorherigen Abschnitten beschriebenen Praktiken und Kriterien auswirken. Darüber hinaus führt die enge Beziehung zwischen Bewertenden und Bewerteten in Nachrufen aber auch dazu, dass sich die Bewertungen nicht nur auf die Verstorbenen beschränken. Autoren von Nachrufen machen sich regelmäßig selbst in ihrer Funktion als Autor sichtbar (zum poststrukturalistischen Konzept der Autorfunktion vgl. Foucault 1977). Nachdem die beiden vorherigen Abschnitte zu den Bewertungspraktiken und -kriterien die Fremdbewertung der Verstorbenen fokussiert haben, widmet sich dieser Abschnitt der Frage, inwiefern durch die Einnahme spezifischer Sprecherrollen durch die Autoren ein anderer Bewertungsmodus zum Zuge kommt - der der Selbstbewertung. Autorinnen können in Nachrufen zwei typische Sprecherrollen einnehmen: Sie treten entweder als persönliche Vertraute auf oder sie positionieren sich als Sprachrohr ihrer Fachgemeinschaft. Inwiefern ermöglichen diese Sprecherrollen Selbstbewertungen?

Wenn Autoren selbst Teil ihrer Erzählung werden, treten sie nicht nur über persönliche Fürwörter oder als „Vf.“ in Erscheinung (siehe dazu Etzemüller 2013). In persönlicher Stimmlage nehmen Erzählerinnen auch Bewertungen mit explizitem Bezug auf ihre eigenen Erfahrungen und Vorstellungen vor. Regelmäßig streuen sie eigene Werturteile ein: ,ich glaube man muss anerkennen, dass“ (Blumer 1967, S. 103); „ich selbst habe [...] miterleben können, wie aufopferungsvoll [der Verstorbene, J.H.] Arbeit für die Sektion geleistet hat“ (Münch 1987, S. 627). Autoren qualifizieren ihr Urteil außerdem mit Bezug auf ihre eigene Position als Schüler (Broszat 1984, S. 689) oder als Student des Verstorbenen (Gollwitzer 1967, S. 315). Weiterhin

\footnotetext{
${ }^{4}$ Ebenso wichtig wie die Frage, welche Kriterien der Bewertung akademischer Lebensleistungen zugrunde liegen, ist die Frage, welche Maßstäbe keine Rolle spielen, um verdienstvolle Leben in der Wissenschaft auszuzeichnen. Die mangelnde Berücksichtigung bestimmter Kategorien wie Geschlecht und Schichtzugehörigkeit, die dezidiert nicht als relevante Bewertungskriterien gelten, habe ich an anderer Stelle thematisiert (Hamann 2016b).
} 
treten Autoren selbst in Erscheinung, wenn sie den persönlichen Kontakt mit dem Verstorbenen thematisieren und z. B. von einem engen Briefkontakt (Gombrich 1981, S. 337) oder einer direkten Nachbarschaft berichten (Liddington 1984, S. 214). So betonen sie, „wie stark die persönlichen Erfahrungen sich geltend machen“(Wehler 1985, S. 151). Schließlich kann auch Vertrautheit symbolisiert werden, etwa wenn Verstorbene - wie es besonders im englischsprachigen Raum vorkommt - bei ihrem Spitznamen genannt werden (Lemert und Piccone 1981, S. 163; D’Haenens 2007, S. 75). Diese Beispiele zeigen, dass subjektive Wertungen, Selbstpositionierungen und der Verweis auf persönliche Vertrautheit nicht nur Mittel sind, um der Fremdbewertung der Verstorbenen Nachdruck zu verleihen. Sie wirken auch als Selbstbewertung der Autorinnen, die auf diese Weise vor ihrer Fachgemeinschaft als enge Vertraute von besonders verdienstvollen Wissenschaftlern erscheinen.

Die beschriebenen persönlichen Einschübe der Autoren erfolgen regelmäßig, sind aber üblicherweise auf bestimmte Textabschnitte begrenzt. Sie werden auf diese Weise getrennt von der Bewertung im Namen eines Kollektivs, einer Sprecherrolle, die in Nachrufen deutlich mehr Raum einnimmt. Hier fungieren die bewertenden Autoren als die „ordnungsgemäß beauftragten Sprecher“, deren Urteile ,,immer das Produkt kollektiver Arbeit“ sind (Bourdieu 1992, S. 379). Autorinnen sprechen dann als Stimme ihrer Schule oder Disziplin. Sie erklären etwa, dass „wir um unseren Wegweiser und Ratgeber trauern“ (Edelstein 1963, S. 451), dass „der Tod einen wundervollen Kollegen aus unserer Mitte gerissen“ hat (Hillerbrand 2001, S. 1098) oder dass ,wir [...] in ihm einen Kollegen [verlieren], der viel für unsere Profession geleistet hat"“ (Münch 1987, S. 625). Kollektive Bewertungen können auch stellvertretend vorgenommen werden, z.B. im Namen einer „Generation“, für die der Verstorbene „Zeitgeschichte personifiziert hat“ (Medlicott 1969, S. 201), im Namen einer „Gilde“, die durch den verfrühten Tod eines Verstorbenen um ein Mitglied beraubt wurde (Hillerbrand 2001, S. 1098), oder wenn Autoren im Namen von „Zeitgenossen urteilen, was er für uns bedeutete“ (Edelstein 1963, S. 456). Im Unterschied zu personalisierten Bewertungen verweisen solche Wertzuschreibungen auf ein Kollektiv, in dem die Verstorbene verortet wird und für das die Autorin zu sprechen in Anspruch nimmt. Auch die Wertung im Namen eines Kollektivs ist mehr als eine Fremdbewertung der Verstorbenen. Der Autor nimmt gleichzeitig eine Selbstbewertung vor, weil er rhetorisch als autorisierter Sprecher einer Fachgemeinschaft auftritt und in ihrem Namen das letzte Urteil über ein verdientes Mitglied der Gruppe fällt.

Die Unterscheidung der Modi Fremdbewertung und Selbstbewertung erlaubt es, personenbezogene Bewertungen in Nachrufen als zweiseitige Konstellationen zu sehen, in denen Wertzuschreibungen in beide Richtungen stattfinden. So geraten Selbstkategorisierungen und 
-legitimierungen als zentrale Bewertungsprozesse in den Blick. Im Rahmen der hier herausgearbeiteten Sprecherrollen treten Autorinnen als (selbst)bewertende Instanzen explizit in Erscheinung. Sie können mit unterschiedlichen Stimmlagen verschiedene Quellen der Bewertungsautorität anzapfen. Fällen Autoren persönliche Urteile, erhalten ihre Bewertungen gerade durch ihre Subjektivität und Nähe zum Bewertungsobjekt ein besonderes Gewicht (zum epistemischen Wert von Subjektivität in Bewertungen vgl. Chong 2013). Sprechen Autoren dagegen als Stellvertreter eines Kollektivs, beanspruchen sie für ihr Urteil eine überindividuelle Geltung, weil sie durch die Gruppe autorisiert zu sein scheinen. Die in Nachrufen vorgenommenen Wertzuschreibungen stellen also nicht rein sachliche oder objektive Urteile über Personen dar. Es ist nicht zuletzt dieser offensichtliche Unterschied zu anderen personenbezogenen akademischen Bewertungen, der einen systematischeren Vergleich von Nachrufen mit anderen Genres der Bewertung von Personen durch Personen als lohnenswert erscheinen lässt.

\section{Genres der personenbezogenen Bewertung im explorativen Vergleich}

Bereits bei der Erörterung der empirischen Ergebnisse des vorherigen Abschnitts hat sich immer wieder gezeigt, dass es fruchtbar ist, die rekonstruierten Praktiken, Kriterien und Modi der Bewertung in akademischen Nachrufen mit anderen Genres der personenbezogenen Bewertung in Beziehung zu setzen. Der folgende Vergleich wird systematischer herausarbeiten, was die Besonderheiten von Bewertungen in Nachrufen sind, wo es Überschneidungen mit und Unterschiede zu anderen Genres der personenbezogenen Bewertung gibt und in welchem Verhältnis akademische Bewertungen zu Bewertungen in anderen Feldern stehen.

Um die Einsichten aus dem vorherigen Abschnitt komparativ zu kontextualisieren, ziehe ich Vergleichsfälle aus anderen akademischen und nicht-akademischen Genres der personenbezogenen Bewertung heran. Als Vergleichsfolie dienen zum einen nicht-akademische personenbezogene Bewertungen in religiösen Beichten (Hahn 1997; Bilstein 2000) und in psychotherapeutischen Gesprächen (Bruder 2010; Fuchs-Heinritz 2009). Zum anderen wird das literarische Genre der Wissenschaftlerbiografie als ein weiteres Beispiel für eine personenbezogene akademische Bewertung herangezogen (Söderqvist 2011; Govoni und Franceschi 2014). Wie ähneln und unterscheiden sich die Bewertungen in Beichten, Therapiegesprächen, Biografien und Nachrufen, und inwiefern schärft dies den Blick für die Besonderheiten von Nachrufen? Ich greife beim Vergleich der vier Genres auf sieben Vergleichsdimensionen zurück: Ob- 
jekt der Bewertung, Bewertungsinstanz, Öffentlichkeit der Bewertung, Grad der Formalisierung, Funktion des Bewertungsprozesses sowie Folgen der Bewertung für die Bewertenden und die Bewerteten (vgl. die Übersicht in Tab. 1).

Um seinen Gegenstand als Objekt der Bewertung zuzurichten, konstruiert das literarische Genre der Wissenschaftlerbiografie aus einem Lebenslauf eine biografische Erzählung. In einer biografischen Erzählung werden Lebensläufe im Allgemeinen sowie einzelne Aspekte eines Lebens im Besonderen danach kategorisiert und gewichtet, ob sie für etwas Besonderes stehen, mit dem sich ein Leben vom Allgemeinen abhebt (etwa in einer Biografie über Alexander von Humboldt), oder ob sie gerade repräsentativ sind für etwas Allgemeines (etwa für das Leben von Arbeiterinnen im England der Industrialisierung). Ein ähnliches Muster der Objektkonstitution ist im Therapiegespräch und in der Beichte zu erkennen. Wie im Genre der Biografie werden in beiden Bewertungsgenres Lebensläufe zugrunde gelegt, die auf einen spezifischen Aspekt hin befragt werden. Während es die entscheidende Frage der Beichte ist, wie sündhafte Handlungen oder Handlungsmotive im Kontext eines gesamten Lebenslaufs einzuordnen sind und ob ein Leben als Ganzes als das eines Erwählten erscheint, dreht sich das psychotherapeutische Gespräch darum, ob und wie pathologische Züge in einer vom Patienten erzählten Lebensgeschichte diagnostiziert werden können. Auch akademische Nachrufe gehen von einem gesamten Lebenslauf aus und konstruieren daraus eine sinnvolle, gerichtete Biografie. Der selektive Blick der Bewertung sucht hier aber nicht nach biografischer Repräsentativität, Besonderheiten, Sünden oder Pathologien, sondern bildet ab, inwiefern ein Leben in der Wissenschaft verdienst- und ehrenvoll war. Dabei werden beispielsweise die oben behandelten Kriterien „Begabung“ und „Internationalität“ mobilisiert, und von besonderer Bedeutung sind Bewertungspraktiken, die Wissenschaftlerinnen durch Kategorisierungen sortieren und ihre Lebensläufe legitimieren.

Die Vergleichsfälle weisen unterschiedliche Bewertungsinstanzen auf, die die Bewertung vornehmen. In der Beichte nimmt die Bewertung der Beichtvater vor, der dem Beichtsohn gegenübersteht und als Stellvertreter Gottes fungiert (im Protestantismus nimmt der Beichtvater dagegen eher die Rolle eines Gesprächspartners ein, der die Selbstreflexion der Einzelnen begleitet). Im Therapiegespräch versuchen Therapeutinnen ein Vertrauensverhältnis zu den Patienten zu entwickeln, mit deren Krankengeschichte sie sich auseinandersetzen. Eine distanziertere Bewertungsinstanz ist im Genre der Wissenschaftlerbiografie zu erkennen. Zwar können Biografien von Schülerinnen oder Familienmitgliedern verfasst werden, in der Regel zeichnen für sie aber Feuilletonisten, Historikerinnen oder professionelle Biografen verantwortlich. Zu- 
meist deutlich näher stehen die Bewertungsinstanzen den Bewerteten in akademischen Nachrufen. Oben habe ich gezeigt, dass die Beziehung zwischen Autoren und Verstorbenen in verschiedenen Sprecherrollen explizit thematisiert wird. Autoren kommen in der Regel aus dem Kreis der Fachkollegen und sprechen demzufolge auch als Stellvertreter dieses Kollektivs. Oft stehen sie in einer engen Beziehung zum Bewertungsobjekt, was die Sprecherrolle der persönlichen Vertrautheit unterstreicht. Während eventuelle Beziehungen zwischen Bewertungsinstanz und -objekt in anderen Genres, z. B. in anonymen Gutachten, aber auch in Biografien, nicht offen gelegt werden, erhält die Bewertung in Nachrufen ihr Gewicht gerade durch die Nähe zwischen Bewertenden und Bewerteten. Weil Autoren von Nachrufen als legitime Sprecher erscheinen, die Verstorbene in angemessener Form und im Namen der Fachgemeinschaft bewerten können, steht hinter dieser personifizierten Bewertungsinstanz - wie auch in der Beichte - eine schwerer zu fassende Institution, die gewissermaßen das Mandat zur Bewertung erteilt hat. In der dyadischen Konstellation der Beichte scheint die Instanz des Beichtvaters von Gott ermächtigt zu sein. In der Bewertungsdyade des Nachrufs tritt der Autor als Sprachrohr einer Fachgemeinschaft auf, die ihm das letzte Urteil anvertraut zu haben scheint.

Die Vergleichsfälle unterscheiden sich auch hinsichtlich der Öffentlichkeit der Bewertung. Auf der einen Seite stehen Bewertungen, die explizit nicht-öffentlich sind. Dazu gehört, bis auf historische Ausnahmen, die Beichte. Das Beichtgeheimnis garantiert, dass die Bewertungskonstellation gegenüber ihrer Außenwelt abgeschlossen bleibt. Interessant ist, dass diesem Moment der Verhüllung gleichzeitig eine Enthüllung im Sinne einer Offenbarung des Beichtenden gegenübersteht. Hier besteht eine Parallele zum ebenfalls nicht-öffentlichen Bewertungsgenre des Therapiegespräches. Wie das Beichtgeheimnis garantiert die ärztliche Schweigepflicht eine Verhüllung der Bewertung. Dies wiederum ermöglicht erst die Selbstenthüllung und Offenbarung des Patienten. Therapiegespräch und Beichte sind also nichtöffentliche Bewertungen, deren Verfahren und Ergebnisse für Personen außerhalb der Dyade von Bewertungsobjekt und -instanz nicht ohne Weiteres einsehbar sind. Die Bewerteten können der Redlichkeit und Verschwiegenheit der bewertenden Instanz in einem hohen Grade vertrauen und sich ihr gerade deshalb auch anvertrauen. Diesen Fällen von Bewertungen, in denen die Bewertungsergebnisse nur für die bewerteten Personen bestimmt sind, stehen öffentliche Bewertungen gegenüber, in denen personenbezogene Bewertungen nicht nur durch ein Publikum einsehbar, sondern für eine Öffentlichkeit geradezu vorgesehen sind. Die Wissenschaftlerbiografie wird für ein breiteres, öffentliches Publikum produziert. Auch Nachrufe bewerten Personen nicht nur vor einem Publikum, sondern können dies sogar in seinem Namen tun. Während sich Biografien jedoch an eine interessierte breitere Öffentlichkeit wenden, adressieren 
Nachrufe den engeren Kreis der Fachöffentlichkeit, die sich je nach Reichweite der veröffentlichenden Fachzeitschrift in der Regel auf spezialisierte Communities beschränkt.

Die Bewertungsgenres variieren auch mit Blick auf ihren Formalisierungsgrad im Sinne einer mehr oder weniger konkret definierten Form der Durchführung der Bewertung. Wissenschaftlerbiografien sind nicht formalisiert, weil sie weder stilistischen noch zeitlichen oder thematischen Vorgaben unterworfen sind. Auch die Bewertungsregeln und -kriterien sind nicht systematisiert. Stark formalisiert sind hingegen die institutionalisierten Bewertungen in der religiösen Beichte. So gibt es zahlreiche historische Beispiele von Handbüchern, die für den Beichtvater jene Sünden, Intentionen und Tugenden katalogisieren, die für das Beichtgespräch relevant sind. Auch zeitlich ist die Beichte formalisiert; von der Häufigkeit bis zum genauen Ablauf ist eine detaillierte „Rhythmisierung der Beicht-Choreographie“ zu erkennen (Bilstein 2000, S. 614). Zwischen den nicht formalisierten Biografien und der stark formalisierten Beichte liegen die moderat formalisierten Bewertungen. Dazu gehören Therapiegespräche, in denen medizinische Systematisierungen von Symptomen oder reglementierte therapeutische Maßnahmen mit unstrukturierten Gesprächen oder formalisierten Techniken der strukturierten Gesprächsführung kombiniert werden. Moderat formalisiert sind auch Nachrufe. Die Länge der Texte ist durch Vorgaben der veröffentlichenden Fachzeitschriften begrenzt. Das reglementiert auch die Ausführlichkeit der vorgenommenen Bewertung. Außerdem folgen viele Nachrufe hinsichtlich der wesentlichen Elemente des Textaufbaus einem Muster. So ermöglicht eine Einleitung mit Geburts- und Sterbedatum sowie der institutionellen Anbindung eine erste Einordnung der Verstorbenen, während ein abschließender Absatz die Verstorbenen noch einmal zusammenfassend bewertet und den Nachruf mit einer Trauerformel beendet. Autorinnen werden für Nachrufe jedoch nicht immer formal angefragt, sondern bieten sich selbst zu Nachrufen über ihnen nahestehende Fachkollegen an.

Die Vergleichsfälle bilden eine große Bandbreite von Funktionen der Bewertung ab. In der Beichte soll ein den Sünden genau angemessenes Strafmaß gefunden werden (der technische Begriff hierfür ist „Tarifbuße“; siehe Bilstein 2000, S. 612). Vor dem Hintergrund ihrer gesamtbiografischen Verantwortung sollen Beichtende zur Einsicht kommen und Reue entwickeln. Damit verwandt ist das Therapiegespräch mit seiner Funktion der Pathogenese, die von der Analyse des Unbewussten über die persönliche Diagnose bis zum medizinischen Befund variieren kann. Beichte und Therapiegespräch dienen damit der Ausübung sozialer Kontrolle, einem Willen zum Wissen, der die Psyche und das Gewissen des Subjekts ausleuchtet (zur medizinisch-wissenschaftlichen Kontrollfunktion der Therapie vgl. Foucault 1983, zur Konstitution und Unterwerfung des Subjekts durch die Beichte Foucault 2014). Bewertungen in Form 
von Wissenschaftlerbiografien haben die Funktion, akademische Lebensläufe zu dokumentieren. Dazu markieren sie Lebensläufe beziehungsweise Aspekte dieser Leben als interessant und wichtig und folgen dem Anspruch, sie möglichst realitätsgetreu abzubilden. Nachrufe haben dagegen eine konsekrierende Funktion und zielen auf eine ehrenvolle Erinnerung akademischer Lebensleistungen. Sie sind demnach das einzige der hier miteinander verglichenen Bewertungsgenres, das explizit nicht an der Enthüllung oder Dokumentation einer Wahrheit interessiert ist, sondern den Autorinnen die literarische Freiheit zugesteht, eine feierliche Ehrung zu produzieren, die bewusst persönliche Probleme, fachliche Kritik oder biografische Brüche ausblendet. Beispielhaft deutlich wird dies in den oben behandelten Bewertungspraktiken der Legitimierung und Anerkennung, die biografische Brüche und fachliche Kritik nachträglich rationalisieren oder externalisieren.

Verbunden mit den diversen Funktionen der Bewertungsgenres sind ihre teils gleichartigen, teils unterschiedlichen Folgen und Effekte für die Bewerteten und die Bewertenden. Wieder ähneln sich Beichte und Therapiegespräch. Für die Bewerteten ist eine Folge nicht nur die mögliche Genesung oder Tugendhaftigkeit, sondern auch eine Subjektivierung von Krankheitssymptomen oder des Schuldempfindens. Für die Bewertenden ist die Folge eine Anerkennung und Reproduktion ihrer Pastoralmacht (Foucault 2005). Das Resultat der Bewertung im Genre der Wissenschaftlerbiografie ist ein linearer, gerichteter Lebenslauf in Form eines biografischen Artefakts (Bourdieu 1998). Während die in der Biografie behandelten Wissenschaftlerinnen konsekriert werden und ihnen allein schon durch die Selektivität des Genres eine sozial verbindliche Weihe zuteilwird, gelten Biografen in der Regel fortan als Experten für die literarisch portraitierte Person. Die Folgen der Bewertung in Nachrufen sind denen im Genre der Biografie ähnlich: Durch die narrative Verknüpfung von Stationen im Lebenslauf entstehen biografische Artefakte, die ein Leben in der Wissenschaft darstellen. Die bewerteten Biografien werden durch Nachrufe ebenfalls konsekriert. In der Folge wird auch kommuniziert, welche Erwartungen im Feld generell an akademische Lebensläufe gestellt werden - erinnert sei nur an die Bewertungskriterien der „Begabung“ und der „Internationalität“. Nachrufe können zudem auch für ihre Autorinnen einen Konsekrationseffekt zeitigen: Vermittelt über den oben behandelten Modus der Selbstbewertung können sie sich als enge Vertraute eines verdienten Wissenschaftlers oder als autorisiertes Sprachrohr ihrer Fachgemeinschaft präsentieren. 
Tab. 1 Nicht-akademische und akademische Genres der personenbezogenen Bewertung (Quelle: Eigene Darstellung)

\begin{tabular}{|c|c|c|c|c|}
\hline & Beichte & Therapie & Biografie & Nachruf \\
\hline Objekt & Sünden & Pathologien & Lebenslauf & Lebensleistung \\
\hline Instanz & Beichtvater/Gott & Therapeutin & Biograf & Peer/Forschungsgemeinschaft \\
\hline Öffentlichkeit & nicht öffentlich & nicht öffentlich & $\begin{array}{l}\text { breite } \\
\text { Öffentlichkeit }\end{array}$ & Fachöffentlichkeit \\
\hline Formalisierung & stark formalisiert & $\begin{array}{l}\text { moderat } \\
\text { formalisiert }\end{array}$ & $\begin{array}{l}\text { nicht } \\
\text { formalisiert }\end{array}$ & moderat formalisiert \\
\hline Funktion & Reue & Pathogenese & Dokumentation & Würdigung \\
\hline $\begin{array}{l}\text { Folgen für } \\
\text { Bewertende }\end{array}$ & $\begin{array}{l}\text { religiöse } \\
\text { Pastoralmacht }\end{array}$ & $\begin{array}{l}\text { weltliche } \\
\text { Pastoralmacht }\end{array}$ & Expertise & Auszeichnung \\
\hline $\begin{array}{l}\text { Folgen für } \\
\text { Bewertete }\end{array}$ & Tugend & Genesung & Kanonisierung & Konsekration \\
\hline
\end{tabular}

Die Übersicht in Tab. 1 fasst die wichtigsten Dimensionen und Merkmale des explorativen Vergleichs zusammen. Mit Blick auf akademische Bewertungen hat der Vergleich, erstens, deutlich gemacht, dass Wertzuschreibungen in der wissenschaftlichen Welt nur lückenhaft behandelt werden, wenn sich die soziologische Forschung vor allem auf nicht-personale Begutachtungen von Projektanträgen oder Manuskripten konzentriert. Erst wenn auch die bisher in der Forschung eher randständigen personenbezogenen Wertzuschreibungen in den Blick genommen werden, erschließt sich die Diversität von Bewertungsobjekten, -funktionen und -folgen sowie von Bewertungskriterien, -praktiken und -modi in der Wissenschaft. Der Vergleich zeigt, zweitens, dass die Charakteristika von Bewertungsgenres nicht einfach von den sozialen bzw. institutionellen Feldern abgeleitet werden können, in denen die Bewertungen stattfinden. Akademische Bewertungen in Nachrufen sind in wichtigen Punkten den religiösen Bewertungen in der Beichte ähnlicher als anderen akademischen Wertzuschreibungen wie z.B. Fachgutachten oder Wissenschaftlerbiografien. Um charakteristische Grundzüge von Bewertungen zu rekonstruieren, sind komparative Untersuchungsdesigns besonders fruchtbar, die gezielt und systematisch Bewertungsgenres verschiedener Felder miteinander vergleichen (vgl. Chong et al. 2017; Hamann und Beljean 2017). 


\section{Diskussion: Nachrufe im Licht einer Soziologie der Bewertung}

Akademische Nachrufe sind ein Genre, in dem Peers die Lebensleistungen ihrer verstorbenen Wegbegleiter, Kolleginnen oder akademischen Lehrer bewerten. Abschließend möchte ich die Ergebnisse meines Beitrages in den allgemeineren Zusammenhang des Forschungsfeldes der Bewertungssoziologie einordnen und an die dort vorhandenen Konzepte zurückbinden. Mein Beitrag hat, erstens, anhand typischer Bewertungspraktiken gezeigt, wie Wissenschaftlerinnen durch Kategorisierungen in wissenschaftlichen und institutionellen Kontexten verortet und sortiert werden, während ihre Lebensläufe trotz biografischer Brüche und fachlicher Kritik eine Anerkennung und Legitimierung erfahren. Zweitens wurden Zuschreibungen von „Begabung“ und „Internationalität““ als Beispiele von Bewertungskriterien angeführt, deren Relevanz zeitlich, national und disziplinär variiert. Drittens konnten mit Fremd- und Selbstbewertungen zwei verschiedene Bewertungsmodi unterschieden werden, die sich aus den beiden typischen Sprecherrollen von Autoren von Nachrufen ergeben.

Wie lassen sich diese Praktiken, Kriterien und Modi der Bewertung an die Diskussion zu einer Soziologie der Bewertung rückbinden? Die in diesem Beitrag herausgearbeiteten Bewertungspraktiken zeigen, erstens, wie Wissenschaftlerinnen in Kontexten spezialisierten wissenschaftlichen Wissens und institutioneller Hierarchien verortet werden. Zur Einordnung dieses Befunds lässt sich Lamonts (2012, S. 6f.) Diskussion von Kategorisierungen als nicht immer klar abgrenzbare Bewertungsprozesse heranziehen. Es handelt sich dabei um - z. B. auch in Rankings beobachtbare - Prozesse, bei denen Bewertungsobjekte zunächst anhand zugeschriebener Eigenschaften beurteilt und dann entlang dieser eingeordnet oder typisiert werden. $\mathrm{Zu}$ diesen Prozessen lassen sich auch jene Praktiken rechnen, anhand derer Wissenschaftler in diverse Kontexte einsortiert werden bzw. sich wechselseitig selbst einordnen. Vor diesem Hintergrund illustrieren wissenschaftliche und institutionelle Referenzkontexte, dass die in den Bewertungspraktiken mobilisierten Kategoriensysteme ineinandergreifen und miteinander verwoben sein können. Kategorisierungen sind trotz dieses enormen Komplexitätsgrades intelligibel, weil die Fachöffentlichkeit zumeist in der Lage ist, die hochgradig differenzierten und ineinander verschachtelten Verweise in den Nachrufen zu dechiffrieren.

Die in diesem Beitrag untersuchten Praktiken zeigen, zweitens, wie biografische Brüche oder fachliche Kritik nachvollziehbar und plausibel in die Gesamterzählung eines erfolgreichen Lebens integriert oder ganz ausgeklammert werden, sodass insgesamt eine positive Gesamtwürdigung des Wissenschaftlers zustande kommt. Lamont (ebd.) bezeichnet solche Prozesse 
der sozial verbindlichen Anerkennung, bei denen Objekte der Bewertung durch Wertzuschreibungen kanonisiert werden, als Prozesse der Legitimierung. In der Bewertungssoziologie ist bisher nicht ausreichend berücksichtigt worden, dass legitimierende Bewertungen nicht nur positive Urteile umfassen. Auch negative Aspekte wie Krisen und Kritik können ein Teil des Legitimationsprozesses sein, sofern die Autoren von Nachrufen die Herausforderung meistern, sie sinnvoll in die Erfolgsgeschichte eines verdienstvollen Lebens zu integrieren. Auf diese Weise erfüllen Nachrufe ihre konsekrierende Bewertungsfunktion.

Einige Kriterien für die Bewertung von akademischen Lebensleistungen variieren je nach ihren disziplinären, zeitlichen und nationalen Kontexten. Dieser Befund erweitert die Sichtweise der Bewertungssoziologie, die Bewertungsprozesse bisher vor allem in ihrer relativ spontanen Situativität untersucht hat. Der Fokus auf die Kreativität und Pluralität von Bewertungspraktiken im Rahmen verschiedener Rechtfertigungsordnungen (vgl. Boltanski und Thévenot 1983; Boltanski und Thèvenot 2006) hat wichtige Impulse gesetzt, es aber weitgehend versäumt, jenen Einflussfaktoren nachzugehen, die die spezifischen Situationen überspannen und vorstrukturieren. Bei akademischen Nachrufen fallen vor allem zwei Institutionen auf, die Bewertungspraktiken in diesem Sinne strukturieren: das wissenschaftliche Feld selbst, von dem beispielsweise wissenschaftsspezifische Bewertungskriterien vorgegeben werden, und die Institution der Pietät, die es z.B. verbietet, in Nachrufen zu negativ über die Verstorbenen zu urteilen. Lamont (2012, S. 11 ff.) erklärt diese Strukturierung von Bewertungskriterien mit alltäglichen Regeln oder Konventionen von Feldern. Der vorliegende Beitrag weist jedoch über die strukturierende Wirkung feldspezifischer Konventionen und Institutionen hinaus. Die Variationen von Bewertungskriterien nach zeitlichen, nationalen und disziplinären Kontexten erinnern daran, dass Bewertungen ebenso von allgemeineren Kontexten gerahmt und strukturiert sein können. Der unterschiedliche Stellenwert, den das Bewertungskriterium der „Begabung“ in verschiedenen Ländern hat und den das Kriterium der „Internationalität“ im Zeitverlauf und bei unterschiedlichen Disziplinen aufweist, lässt auf den Einfluss weiterer sozialer und kultureller Kontexte auf Bewertungen schließen. Es ist zu vermuten, dass sich der Einfluss diverser Kontexte stärker in Bewertungsgenres niederschlagen kann, die, wie Nachrufe, einen geringen Formalisierungsgrad aufweisen. Spiegelbildlich lässt die starke Formalisierung der Beichte erwarten, dass in diesem Fall die nationalen oder zeitlichen Variationen geringer ausgeprägt sind. Die vergleichsweise hohe historische Stabilität der Beichte lässt diese Vermutung zumindest nicht als abwegig erscheinen (vgl. Hahn 1997).

Die in dem Beitrag vorgeschlagene Differenzierung zwischen den Modi der Fremd- und Selbstbewertung verdeutlicht, dass nicht in jeder Bewertungskonstellation eindeutig definiert 
ist, welches Objekt eigentlich der Gegenstand der Bewertung ist, woran die Wertzuschreibung genau orientiert sein soll oder wie sich verschiedene Bewertungskriterien zueinander verhalten.

Dies greift Überlegungen zu Heterarchie oder Pluralitäten in Bewertungskonstellationen auf, z.B. in Form nebeneinander existierender Kriterien oder Hierarchien zwischen den Bewertungskriterien (Lamont 2012, S. 7 ff.). Die explorativen empirischen Befunde dieses Beitrages lassen sich an diese Überlegungen rückbinden: Erstens legt der Vergleich von Nachrufen mit anderen Genres personenbezogener Bewertungen nahe, dass die Vielfalt der in den Nachrufen mobilisierten Bewertungskriterien auf den geringen Grad der Formalisierung des Genres zurückgeführt werden kann. Bewertungsgenres, die stärker formalisiert sind als Nachrufe, zeichnen sich demzufolge durch eine eindeutigere Vorgabe und Hierarchie von Kriterien aus. Zweitens zeigen die Befunde, dass sich nicht nur die Kriterien von Bewertungen durch eine Pluralität auszeichnen (vgl. dazu Boltanski und Thèvenot 2006), sondern gleichermaßen nach unterschiedlichen Bewertungsinstanzen zu fragen ist, die gewissermaßen nebeneinander operieren. Das Beispiel der personenbezogenen Bewertung in akademischen Nachrufen zeigt zudem, dass diese Form von Bewertung nicht nur als Fremdbewertung zu verstehen ist. Sie stellt in einem weiteren Sinne auch eine Selbstbewertung dar, die die Bewertungskompetenz einer Bewertungsinstanz beglaubigt und auf diese Instanz zurückwirkt.

\section{Literatur}

Abbott, A. (1999). Department and discipline. Chicago Sociology at one hundred. Chicago: Chicago University Press.

Alexander von Humboldt-Stiftung (2009). Publikationsverhalten in unterschiedlichen wissenschaftlichen Disziplinen. Beiträge zur Beurteilung von Forschungsleistungen. Bonn: Alexander von Humboldt-Stiftung.

Angermuller, J. (2013). How to become an academic philosopher. Academic discourse as multileveled positioning practice. Sociología histórica, (2), 263-289.

Angermuller, J. (2014). Poststructuralist discourse analysis. Subjectivity in enunciative pragmatics. Basingstoke: Palgrave Macmillan.

Baert, P. (2011). The sudden rise of French existentialism: A case-study in the sociology of intellectual life. Theory and Society, 40, 619-644.

Bamberg, M.G.W. (2011). Who am I? Narration and its contribution to self and identity. Theory \& Psychology, 21, 1-22.

Beaufaÿs, S. (2003). Wie werden Wissenschaftler gemacht? Beobachtungen zur wechselseitigen Konstitution von Geschlecht und Wissenschaft. Bielefeld: transcript.

Becher, T., \& Trowler, P. (2001). Academic tribes and territories: Intellectual enquiry and the cultures of disciplines. Philadelphia: Open University Press.

Bilstein, J. (2000). Die Beichte und ihre Bedeutung im Sozialisationsprozess. Zeitschrift für Erziehungswissenschaft, 3, 609-628. 
Boltanski, L., \& Thévenot, L. (1983). Finding one's way in social space: A study based on games. Social Science Information, 22, 631-680.

Boltanski, L., \& Thèvenot, L. (2006). On justification: Economies of worth. Princeton: Princeton University Press.

Bonsu, S. K. (2007). The presentation of dead selves in everyday life: Obituaries and impression management. Symbolic Interaction, 30, 199-219.

Bornmann, L., \& Daniel, H.-D. (2005). Selection of research fellowship recipients by committee peer review: Analysis of reliability, fairness and predictive validity of board of trustees' decisions. Scientometrics, 63, 297-320.

Bourdieu, P. (1992). Homo academicus. Frankfurt a.M.: Suhrkamp.

Bourdieu, P. (1998). Die biographische Illusion. In P. Bourdieu, Praktische Vernunft. Zur Theorie des Handelns (S. 75-90). Frankfurt a.M.: Suhrkamp.

Bruder, K.-J. (2010). Die Kontinuität des bewussten Diskurses - biographisches Interview und psychoanalytisches Gespräch. In B. Griese (Hrsg.), Subjekt - Identität - Person? Reflexionen zur Biographieforschung (S. 73-92). Wiesbaden: VS Verlag für Sozialwissenschaften.

Chong, P. (2013). Legitimate judgment in art, the scientific world reversed? Maintaining critical distance in evaluation. Social Studies of Science, 43, 265-281.

Chong, P., Lamont, M., \& Bourgoin, A. (2017). The blackboxing of expert judgment: Fiction reviewing, scholarly evaluation and management consulting. Manuskript.

Dávidházi, P. (Hrsg.). (2014). New publication cultures in the humanities. Exploring the paradigm shift. Amsterdam: Amsterdam University Press.

Dietz, J. S., Chompalov, I., Bozeman, B., Lane, E.O.N., \& Park, J. (2000). Using the curriculum vita to study the career paths of scientists and engineers: An exploratory assessment. Scientometrics, 49, 419-422.

Echterhölter, A. (2012). Schattengefechte. Genealogische Praktiken in Nachrufen auf Naturwissenschaftler (1710-1860). Göttingen: Wallstein.

Emmel, N. (2013). Sampling and choosing cases in qualitative research. Los Angeles: SAGE.

Etzemüller, T. (2013). Der „Vf.“ als Subjektform. Wie wird man zum „Wissenschaftler“ und (wie) lässt sich das beobachten? In T. Alkemeyer, G. Budde \& D. Freist (Hrsg.), SelbstBildungen. Soziale und kulturelle Praktiken der Subjektivierung (S. 175-196). Bielefeld: transcript.

Foucault, M. (1977). What is an author? In M. Foucault, Language, counter-memory, practice: Selected essays and interviews (S. 113-138). New York: Cornell University Press.

Foucault, M. (1983). Der Wille zum Wissen. Sexualität und Wahrheit, Bd. 1. Frankfurt a.M.: Suhrkamp.

Foucault, M. (2005). Analytik der Macht. Frankfurt a.M.: Suhrkamp.

Foucault, M. (2014). Die Regierung der Lebenden - Vorlesungen am Collège de France 19791980. Frankfurt a.M.: Suhrkamp.

Fowler, B. (2007). The obituary as a collective memory. New York: Routledge.

Fowler, B., \& Bielsa, E. (2007). The lives we choose to remember: A quantitative analysis of newspaper obituaries. The Sociological Review, 55, 203-226.

Fuchs-Heinritz, W. (2009). Biographische Forschung. Eine Einführung in Praxis und Methoden. Wiesbaden: VS Verlag für Sozialwissenschaften.

Gieryn, T.F. (1999). Cultural boundaries of science: Credibility on the line. Chicago: University of Chicago Press.

Gispen, K. (1994). Die deutsche Ingenieurelite, 1840-1930: Eine Analyse der Nachrufe. In P. Lundgreen \& A. Grelon (Hrsg.), Ingenieure in Deutschland, 1770-1990 (S. 221-241). Frankfurt a.M.: Campus. 
Govoni, P., \& Franceschi, Z. A. (Hrsg.). (2014). Writing about lives in science. (Auto)Biography, gender, and genre. Göttingen: Vandenhoeck \& Ruprecht.

Gross, N. (2002). Becoming a pragmatist philosopher: Status, self-concept, and intellectual choice. American Sociological Review, 67, 52-76.

Guetzkow, J., Lamont, M., \& Mallard, G. (2004). What is originality in the humanities and the social sciences? American Sociological Review, 69, 190-212.

Hahn, A. (1997). Zur Soziologie der Beichte und anderer Formen institutionalisierter Bekenntnisse: Selbstthematisierung und Zivilisationsprozess. In J. Friedrichs, K.U. Mayer \& W. Schluchter (Hrsg.), Soziologische Theorie und Empirie (S. 150-177). Opladen: Westdeutscher Verlag.

Hamann, J. (2014). Die Bildung der Geisteswissenschaften. Zur Genese einer sozialen Konstruktion zwischen Diskurs und Feld. Konstanz: UVK.

Hamann, J. (2016). „Let us salute one of our kind“. How academic obituaries consecrate research biographies. Poetics, 56, 1-14.

Hamann, J. (2017). Wie entstehen wissenschaftliche Subjekte? Zum professoralen Ethos akademischer Lebenspraxis. In J. Hamann, J. Maeße, V. Gengnagel \& A. Hirschfeld (Hrsg.), Macht in Wissenschaft und Gesellschaft. Diskurs- und feldanalytische Perspektiven (S. 83-111). Wiesbaden: VS Verlag für Sozialwissenschaften.

Hamann, J., \& Beljean, S. (2017). Gatekeeping in cultural fields. How evaluation processes contribute to social inequality. Manuskript.

Hamann, J., \& Zimmer, L.M. (2017). The internationality imperative in academia. The ascend of internationality as an academic virtue. Higher Education Research \& Development, im Erscheinen.

Hirschauer, S. (2005). Publizierte Fachurteile. Lektüre und Bewertungspraxis im Peer Review. Soziale Systeme, 11, 52-82.

Hornbostel, S. (1997). Wissenschaftsindikatoren. Bewertungen in der Wissenschaft. Opladen: Westdeutscher Verlag.

Huang, F., Finkelstein, M., \& Rostan, M. (Hrsg.). (2014). The internationalization of the academy. Changes, realities and prospects. Dordrecht: Springer.

Huutoniemi, K. (2012). Communicating and compromising on disciplinary expertise in the peer review of research proposals. Social Studies of Science, 42, 897-921.

Lamont, M. (1987). How to become a dominant French philosopher: The case of Jacques Derrida. The American Journal of Sociology, 93, 584-622.

Lamont, M. (2009). How professors think. Inside the curious world of academic judgement. Cambridge: Harvard University Press.

Lamont, M. (2012). Toward a comparative sociology of valuation and evaluation. Annual Review of Sociology, 38, 201-221.

Lewis, L. S. (1998). Scaling the ivory tower: Merit and its limits in academic careers. New Brunswick: Transaction Publishers.

Long, G.L. (1987). Organizations and identity: Obituaries 1856-1972. Social Forces, 65, 9641001.

Macfarlane, B., \& Chan, R.Y. (2014). The last judgement: Exploring intellectual leadership in higher education through academic obituaries. Studies in Higher Education, 39, 294-306.

Maeße, J. (2015). Eliteökonomen. Wissenschaft im Wandel der Gesellschaft. Wiesbaden: VS Verlag für Sozialwissenschaften.

McNamee, S. J., \& Miller, R.K. J. (2004). The meritocracy myth. Langham: Rowman \& Littlefield.

Miller, N., \& Morgan, D. (1993). Called to account: The CV as an autobiographical practice. Sociology, 27, 133-143.

Musselin, C. (2009). The market for academics. New York: Routledge. 
Patton, M.Q. (2002). Qualitative research \& evaluation methods. Thousand Oaks: SAGE.

Reinhart, M. (2012). Soziologie und Epistemologie des Peer Review. Baden-Baden: Nomos.

Rodler, C., Kirchler, E., \& Hölzl, E. (2001). Gender stereotypes of leaders: An analysis of the contents of obituaries from 1974 to 1998. Sex Roles, 45, 827-843.

Sandström, U., \& Hällsten, M. (2008). Persistent nepotism in peer-review. Scientometrics, 74, 175-189.

Sauder, M., \& Espeland, W. N. (2009). The discipline of rankings: Tight coupling and organizational change. American Sociological Review, 74, 63-82.

Söderqvist, T. (2011). The seven sisters: Subgenres of bioi of contemporary life scientists. Journal of the History of Biology, 44, 633-650.

Strauss, A.L., \& Corbin, J.M. (1990). Basics of qualitative research. Grounded theory procedures and techniques. Newbury Park: SAGE.

Tight, M. (2008). Dead academics: What can we learn about academic work and life from obituaries? London Review of Education, 6, 125-135.

Tsay, A., Lamont, M., Abbott, A., \& Guetzkow, J. (2003). From character to intellect: Changing conceptions of merit in the social sciences and humanities, 1951-1971. Poetics, 31, 23-49.

Zimmermann, K. (2000). Spiele mit der Macht. Passfähigkeit und Geschlecht als Kriterien für Berufungen. Berlin: Edition Sigma.

\section{Im Text zitierte Nachrufe}

Blumer, H. (1967). Ernest Watson Burgess, 1886-1966. The American Sociologist, 2, 103104.

Bonnell, V. (1991). In memory of Reinhard Bendix. Berkeley Journal of Sociology, 36, I-V.

Broszat, M. (1984). Nachruf auf Theodor Schieder. Vierteljahreshefte für Zeitgeschichte, 32, 689-690.

Brüche, E. (1960). Abschied von Max von Laue. Physikalische Blätter, 5, 257-258.

Close, F. E., \& Gal, A. (2006). Obituary: Richard Henry Dalitz, FRS (1925-2006). Nuclear Physics, 771, 3-7.

D’Haenens, I. J. (2007). Theodore Harold Maiman. Physics Today, 60(10), 72-75.

Edelstein, L. (1963). In memory of A.O. Lovejoy. Journal of the History of Ideas, 24, 451456.

Foster, R. (2013). Eric Hobsbawm. Past \& Present, 218, 3-15.

Fouquet, G. (2000). Edith Ennen (1907-1999). Vierteljahrschrift für Sozial- und Wirtschaftsgeschichte, 87, 1-4.

Gollwitzer, H. (1967). Karl Alexander von Müller 1882-1964: Ein Nachruf. Historische Zeitschrift, 205, 295-322.

Gombrich, E. H. (1981). In memory of George Boas. Journal of the History of Ideas, 42, 334354.

Hargens, L. L., \& Gieryn, T. F. (1988). Nicholas C. Mullins (1939-1988). Social Studies of Science, 18, 572-573.

Hillerbrand, H. J. (2001). In Memoriam: Bodo Nischan (1939-2001). The Sixteenth Century Journal, 32, 1097-1098.

Kellenbenz, H. (1969). Götz Freiherr von Pölnitz. Vierteljahrschrift für Sozial- und Wirtschaftsgeschichte, 56, 282-288. 
Kopal, Z. (1970). Sydney Chapman (1888-1970). Astrophysics and Space Science, 8, 147148.

Kopal, Z. (1972). In Memoriam Harlow Shapley. Astrophysics and Space Science, 18, 259266.

Lemert, C., \& Piccone, P. (1981). Alvin Ward Gouldner: 1920-1980. Theory and Society, 10, $163-167$.

Liddington, J. (1984). Gloden Dallas (1943-1983). History Workshop Journal, 17, 213-214.

Luckmann, T. (1961). Nekrolog: Alfred Schütz. Kölner Zeitschrift für Soziologie und Sozialpsychologie, 13, 768-770.

Lynch, M. (2011). Harold Garfinkel (29 October 1917 - 21 April 2011): A remembrance and a reminder. Social Studies of Science, 41, 927-942.

Medlicott, W. N. (1969). G.P. Gooch. Journal of Contemporary History, 4, 201-203.

Morris, J. (1970). A. H. M. Jones. Past \& Present, 47, 147-150.

Münch, R. (1987). In memoriam Hans Haferkamp (15.09.1939 - 14.07.1987). Kölner Zeitschrift für Soziologie und Sozialpsychologie, 39, 625-628.

Peacock, N. J., \& Christiansen, J. P. (2005). Rendel Sebastian Pease. Physics Today, 58(6), 78-79.

Rehberg, K.-S. (2003). In memoriam Erwin K. Scheuch (28.6.1928-12.10.2003). Kölner Zeitschrift für Soziologie und Sozialpsychologie, 55, 819-821.

Richards, H. T. (1962). T. W. Bonner (1910-1961). An obituary. Nuclear Physics, 32, 1-4.

Roberts, I. (2007). In Memorium: Richard Kemp Brown. Work, Employment \& Society, 21, 613-614.

Stein, G. (1973). In Memoriam J.J. Weiss (1905-1972). International Journal of Radiation Physics and Chemistry, 5, 137-139.

Wehler, H.-U. (1985). Nachruf auf Theodor Schieder: 11. April 1908 - 8. Oktober 1984. Geschichte und Gesellschaft, 11, 143-153. 